\title{
Compressive sampling for accelerometer signals in structural health monitoring
}

Structural Health Monitoring $0(0) \mathrm{I}-12$

(C) The Author(s) 2010

Reprints and permissions:

sagepub.co.uk/journalsPermissions.nav DOI: $10.1177 / 1475921710373287$ shm.sagepub.com

(S)AGE

\author{
Yuequan Bao ${ }^{1,2}$, James L Beck ${ }^{2}$ and $H u i \mathrm{Li}^{1}$
}

\begin{abstract}
In structural health monitoring (SHM) of civil structures, data compression is often needed to reduce the cost of data transfer and storage, because of the large volumes of sensor data generated from the monitoring system. The traditional framework for data compression is to first sample the full signal and, then to compress it. Recently, a new data compression method named compressive sampling (CS) that can acquire the data directly in compressed form by using special sensors has been presented. In this article, the potential of CS for data compression of vibration data is investigated using simulation of the CS sensor algorithm. For reconstruction of the signal, both wavelet and Fourier orthogonal bases are examined. The acceleration data collected from the SHM system of Shandong Binzhou Yellow River Highway Bridge is used to analyze the data compression ability of CS. For comparison, both the wavelet-based and Huffman coding methods are employed to compress the data. The results show that the values of compression ratios achieved using CS are not high, because the vibration data used in SHM of civil structures are not naturally sparse in the chosen bases.
\end{abstract}

\section{Keywords}

compressive sampling, data compression, structural health monitoring, wavelet, transform, Huffman coding

\section{Introduction}

In the recent years, structural health monitoring (SHM) systems have been installed on civil structures, because they have the ability to observe damage evolution and enhance safety. A complete SHM system may include: sensors, data acquisition system, data transfer and storage mechanisms, data management and data analysis for system identification, structural damage detection, and prediction of remaining service life. Generally, a substantial number of sensors are required in SHM systems, because of the large scale and complexity of civil structures. For example, the sensor system of the Wind and Structural Health Monitoring System (WASHMS) installed on Tsing Ma, Ting Kau, and Kap Shui Mun bridges in Hong Kong, China, consists of approximately 800 sensors. ${ }^{1}$ The structural behavior of the bridges is measured $24 \mathrm{~h}$ a day, 7 days a week, so enormous amounts of sensor data are generated by the WASHMS. Data compression has, therefore, a potentially important role in SHM to save the cost of data transfer and storage.

For SHM of civil structures, data compression has attracted the interest of researchers in the recent years.
Zhang and $\mathrm{Li}^{2}$ presented a data compression method based on the lifting-scheme wavelet transform that can achieve a high compression ratio (CR). Wireless sensor networks for SHM have been investigated, because they not only provide the same functionality at a much lower installation price, but can also process data autonomously by embedded software. ${ }^{3,4}$ Data compression techniques can be used to minimize bandwidth and power consumption during wireless communications so that the lifetime of the wireless sensors can be maximized. Data compression is therefore a crucial aspect of wireless sensor networks. Xu et al. ${ }^{5}$ investigated the applicability of wavelet-based compression techniques to overcome the bandwidth limitations of wireless sensors. Lynch et al. ${ }^{6}$ used Huffman coding

\footnotetext{
'School of Civil Engineering, Harbin Institute of Technology, Harbin 150090, People's Republic of China.

${ }^{2}$ Division of Engineering and Applied Science, California Institute of Technology, Pasadena, CA 9II25, USA.
}

\section{Corresponding author:}

James L Beck, Division of Engineering and Applied Science, California Institute of Technology, Pasadena, CA 9II25, USA

Email: jimbeck@caltech.edu 
to reduce the size of the wirelessly-transmitted data packets. In the past two decades, data compression techniques have also been researched to compress seismic data. ${ }^{7-9}$ However, all these methods belong to a traditional data compression framework.

Recently, a new data compression method called compressive sampling (CS) has been introduced. ${ }^{10-14}$ CS is an alternative theory to Nyquist/Shannon sampling theory that can reconstruct images or signals accurately from far fewer measurements than what is usually considered necessary. ${ }^{10}$ Candès ${ }^{10}$ suggests that this new technique may come to underlie procedures for sampling and compressing data simultaneously. In this article, the application of CS for compression of vibration data is investigated. The acceleration data collected from the SHM system of Shandong Binzhou Yellow River Highway Bridge is employed to investigate the data compression ability of CS.

\section{Compressive sampling}

For more detailed information on CS, see references Candès, ${ }^{10}$ Candès and Tao, ${ }^{11}$ Candès et al., ${ }^{12}$ Donoho, ${ }^{13}$ and Baraniuk ${ }^{14}$ and additional resources can be found at http://dsp.rice.edu/cs/. CS is a new technique, which is still being explored, although some applications have been developed already, such as the CS camera, ${ }^{15}$ compressive wireless sensing, ${ }^{16}$ and CS analog-to-digital converter (ADC). ${ }^{17}$ Only a short introduction to CS is presented here.

Any discrete-time signal $x(t), t=1, \ldots, n$, in $\mathbb{R}$ can be represented in terms of an orthonormal basis of $n \times 1$ vectors $\left\{\psi_{i}\right\}_{i=1}^{n}$. Introducing the $n \times n$ basis matrix $\boldsymbol{\Psi}=\left[\psi_{1}, \psi_{2}, \ldots, \psi_{n}\right]$ with the basis vectors $\psi_{i}$ as columns, then $\boldsymbol{\Psi}^{-1}=\boldsymbol{\Psi}^{T}$ and the vector $x=[x(1), \cdots, x(n)]^{T} \in \mathbb{R}^{n}$ of $n$ sampled values of $x(t)$ can be expressed as:

$$
x=\sum_{j=1}^{n} \alpha_{j} \psi_{j}, \quad \text { or } x=\boldsymbol{\Psi} \alpha
$$

where the basis, or weighting, coefficients $\alpha_{j}$ are given by $\alpha=\boldsymbol{\Psi}^{T} x$.

Suppose that we acquire an $m(<n)$ length data vector $y$ by a linear projection of $x$ :

$$
y=\boldsymbol{\Phi} x=\boldsymbol{\Phi} \boldsymbol{\Psi} \alpha=\boldsymbol{\Theta} \alpha
$$

where $\boldsymbol{\Phi}$ is called a measurement matrix and $\boldsymbol{\Theta}=$ $\boldsymbol{\Phi} \boldsymbol{\Psi}$ an $m \times n$ matrix. Since $\boldsymbol{\Phi}$ is an $m \times n$ matrix with $m<n$, the problem of recovering the signal $x$, which has $n$ components, from the $m$ measurements in $y$, is illposed. CS exploits the fact that if $x$ is known to be sparse in the basis $\boldsymbol{\Psi}$, that is, many of the coefficients $\alpha_{j}$ are zero, then under certain conditions $\alpha$ can be reconstructed exactly by solving the convex optimization:

$$
\text { (P1) } \hat{\alpha}=\arg \min \|\tilde{\alpha}\|_{1} \text { such that } \Theta \tilde{\alpha}=y
$$

The solution $\hat{\alpha}$ can recover $\alpha$ exactly under the following conditions:

1. the signal $x$ is $s$-sparse where a vector is defined to be $s$-sparse if it has at most $s$ nonzero entries;

2. the matrix $\Theta$ obeys a so-called restricted isometry property (RIP), ${ }^{11,12}$ that is, there exists an isometry constant $\delta_{s}$ for the matrix $\boldsymbol{\Theta}$, where $\delta_{s}$ is defined as the smallest number such that:

$$
\left(1-\delta_{s}\right)\left\|v_{s}\right\|_{2}^{2} \leq\left\|\boldsymbol{\Theta} v_{s}\right\|_{2}^{2} \leq\left(1+\delta_{s}\right)\left\|v_{s}\right\|_{2}^{2}
$$

holds for all $s$-sparse vectors $v_{s}$.

Thus, if $\Theta$ satisfies Equation (4), then the signals that are $s$-sparse can be exactly recovered by the optimization problem (P1).

Suppose that $\boldsymbol{\Psi}$ is an orthonormal basis such as a wavelet basis or Fourier sinusoids basis, then we wish to construct a measurement matrix $\boldsymbol{\Phi}$ such that $\boldsymbol{\Theta}=\boldsymbol{\Phi} \boldsymbol{\Psi}$ has the RIP. In CS, $\boldsymbol{\Phi}$ is selected as a random matrix; for example, some matrices are given in Candès, ${ }^{10}$ Candès and Tao, ${ }^{11}$ Candès et al., ${ }^{12}$ and Baraniuk et al. ${ }^{18}$ such as the $m \times n$ matrix $\boldsymbol{\Phi}$ formed by sampling independent and identically distributed entries $\phi_{i, j}$ from either the Normal distribution with mean zero and variance $1 / m$ or the symmetric Bernoulli distribution $\left(P\left(\phi_{i, j}= \pm / \sqrt{m}\right)=1 / 2\right)$. With overwhelming probability, these random Gaussian and Bernoulli matrices $\boldsymbol{\Phi}$ obey the RIP provided that:

$$
m \geq c \cdot s / \log (n / s)
$$

where $c$ is a specified constant. ${ }^{12,13,18}$ The matrix $\boldsymbol{\Theta}=\boldsymbol{\Phi} \boldsymbol{\Psi}$ then also obeys the RIP with $m \geq c \cdot s / \log (n / s)$ regardless of the orthonormal basis matrix $\boldsymbol{\Psi},{ }^{10,14}$ that is, independently of the choice of the transform method. Therefore, given an $s$-sparse signal $x$ of length $n$, as long as the CR $n / m$ does not exceed $c^{-1}(n / s) \log (n / s)$, perfect reconstruction of $x$ is possible from the $m$ measurements in $y$. Here, $n / s$ is a measure of the sparsity of the signal.

Since measurements are usually contaminated by noise, consider the model:

$$
y=\boldsymbol{\Phi} x+e
$$

where $e$ is a bounded but uncertain error with $\|e\|_{2} \leq \varepsilon$. The basis coefficients $\alpha$ can be reconstructed by solving the convex optimization problem:

$$
\text { (P2) } \hat{\alpha}=\arg \min \|\tilde{\alpha}\|_{1} \text { such that }\|\boldsymbol{\Theta} \tilde{\alpha}-y\|_{2} \leq \varepsilon
$$


where $\varepsilon$ is the chosen bound on the size of the measurement error. As for problem (P1) in Equation (3), problem (P2) also has a unique solution and the optimal basis coefficients $\hat{\alpha}$ may be used to represent the data. Now exact recovery of the original signal $x$ is not guaranteed, of course, because the unknown noise is only assumed to satisfy a bound on its norm.

\section{Wavelet de-noising and data compression}

Wavelet multi-resolution analysis (WMRA) is a technique used to perform the discrete wavelet transform. It allows the decomposition of signals into various resolution levels as shown in the Figure 1 where $x, a$, and $d$ are the signals, the low-frequency components called the approximations, and the high-frequency components called the details, respectively.

If the decomposition is stopped at the $J$-th resolution level, the original discrete signal can be approximately reconstructed using the approximation at the $J$-th level and all the details starting from the first level up to the $J$-th level as:

$$
x(n)=\sum_{k} a_{J, k} \cdot \phi_{J, k}(n)+\sum_{j=1}^{J} \sum_{k} d_{j, k} \cdot \psi_{j, k}(n)
$$

where $j$ and $k$ are the scaling and shifting indices, respectively, for the wavelet basis functions. The first term and the second term represent the approximation and the sum of the details up to level $J$, respectively. The approximation coefficient $a_{j, k}$ and details coefficient $d_{j, k}$ are given by:

$$
\begin{gathered}
a_{j, k}=\left\langle x, \phi_{j, k}\right\rangle=2^{(-j / 2)} \sum_{n} x(n) \phi\left(2^{-j} n-k\right) \\
d_{j, k}=\left\langle x, \psi_{j, k}\right\rangle=2^{(-j / 2)} \sum_{n} x(n) \psi\left(2^{-j} n-k\right)
\end{gathered}
$$

where $\phi_{j, k}$ and $\psi_{j, k}$ are the scaling function and wavelet basis functions, respectively. Detailed expositions of wavelets can be found in Daubechies, ${ }^{19}$ Meyer, ${ }^{20}$ and Mallat. $^{21}$

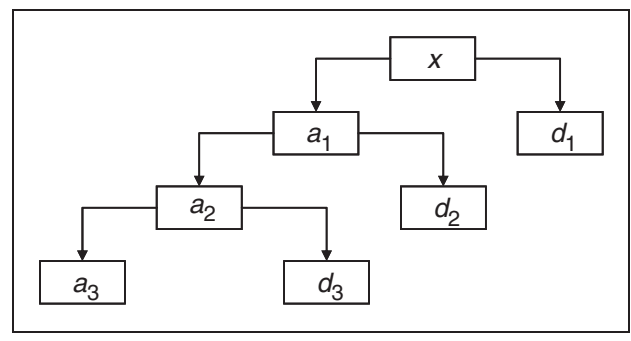

Figure I. Wavelet decomposition tree.
For wavelet de-noising, thresholded wavelet coefficients are used for reconstruction of the data. For wavelet data compression, only the nonzero thresholded wavelet coefficients and their locations are recorded and transmitted. The thresholded wavelet coefficients can be obtained using either the 'hard' or 'soft' thresholding rule given respectively by:

$$
\begin{gathered}
\eta^{H}(\beta, \lambda)= \begin{cases}\beta & |\beta| \geq \lambda \\
0 & |\beta|<\lambda\end{cases} \\
\eta^{S}(\beta, \lambda)=\left\{\begin{array}{cc}
\operatorname{sign}(\beta)(|\beta|-\lambda) & |\beta| \geq \lambda \\
0 & |\beta|<\lambda
\end{array}\right.
\end{gathered}
$$

where $\lambda$ is the threshold level and $\beta$ a wavelet coefficient (either $a_{j, k}$ or $d_{j, k}$ ). Hard thresholding is a 'keep' or 'kill' rule, while soft thresholding is a 'shrink' or 'kill' rule. The threshold level can be calculated from: ${ }^{22}$

$$
\lambda=\hat{\sigma} \sqrt{2 \log n}
$$

where $n$ is the discrete signal length and $\hat{\sigma}$ the noise level estimate: $^{22}$

$$
\hat{\sigma}=\frac{1}{0.6745} \operatorname{median}\left\{\left|d_{j, k}\right|: j=J, k=0,1, \ldots, 2^{J}-1\right\}
$$

involving the median absolute deviation of the wavelet coefficients at the finest level $J$.

\section{Compressive sampling of accelerometer signals from a bridge SHM system}

Shandong Binzhou Yellow River Highway Bridge is a cable-stayed bridge with three towers located in Shandong, China (Figure 2). It has a total length of $768 \mathrm{~m}$, consisting of two $300 \mathrm{~m}$ main spans and two $84 \mathrm{~m}$ side spans. The main tower is $125.28 \mathrm{~m}$ high and the side tower is $75.78 \mathrm{~m}$ high. The bridge was constructed in 2002 and opened to traffic in 2004. A SHM system on this bridge has been operating since 2004. Figure 3 shows the locations of the accelerometers placed on one-half of the bridge. More details of the SHM system can be found in Li et al. ${ }^{23}$

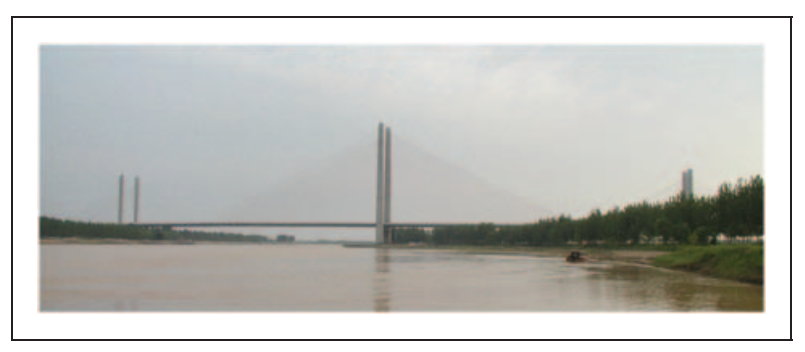

Figure 2. Shandong Binzhou Yellow River Highway Bridge. 


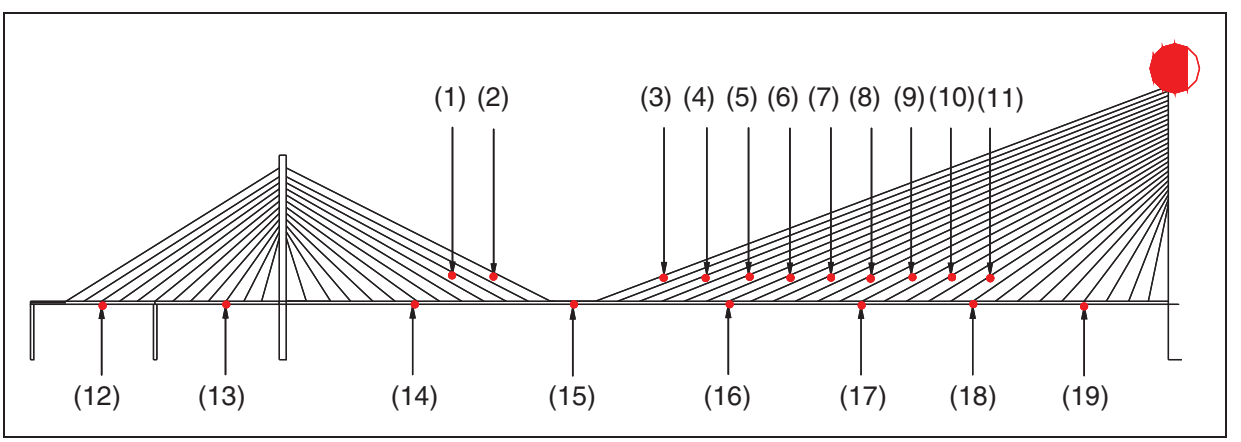

Figure 3. Locations of the accelerometers.

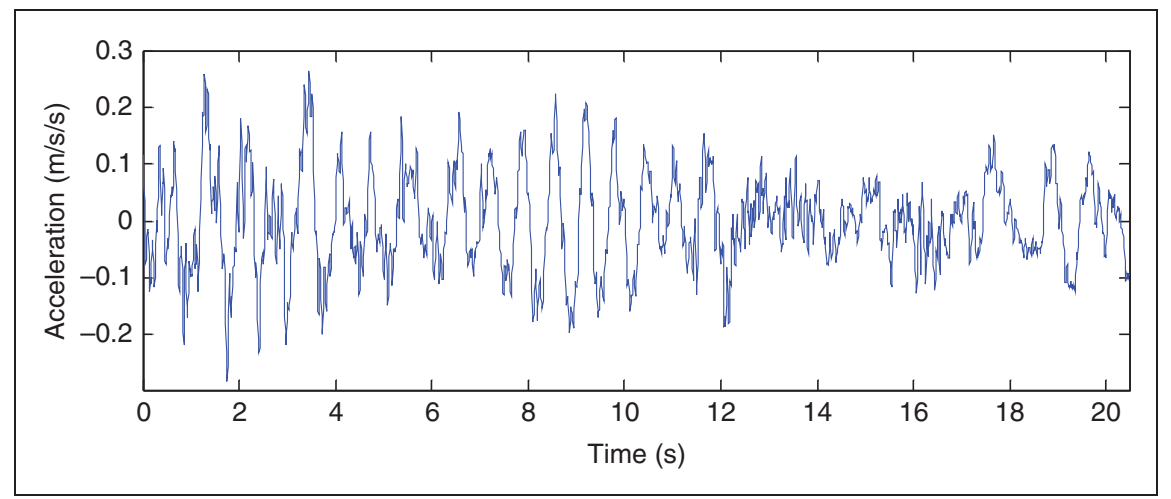

Figure 4. Acceleration response of bridge deck measured by accelerometer 13.

Figure 4 shows the acceleration response of the bridge deck from one accelerometer of this system. There are 2048 samples at a sample frequency of $100 \mathrm{~Hz}$.

The wavelet coefficients of the acceleration data, when decomposed by the Haar wavelet basis with five resolution levels, are shown in Figure 5(a). It can be seen that the wavelet coefficients are not sparse. After using soft threshold de-noising, the wavelet coefficients shown in Figure 5(b) are sparse in the Haar wavelet basis domain because only $s=363$ of them are nonzero, that is, $82.28 \%$ of the $n=2048$ coefficients are zero.

Denoting the de-noised wavelet coefficients by $\alpha_{d}$, the original acceleration can be expressed as:

$$
x(t)=\boldsymbol{\Psi} \alpha_{d}+\tilde{e}
$$

where $\boldsymbol{\Psi}$ is the Haar wavelet basis matrix and $\tilde{e}$ the noise. The measured data can be expressed as:

$$
y=\boldsymbol{\Phi} x=\boldsymbol{\Phi} x_{d}+\boldsymbol{\Phi} \tilde{e}=\boldsymbol{\Phi} \boldsymbol{\Psi} \alpha_{d}+e
$$

where $x$ and $x_{d}=\boldsymbol{\Psi} \alpha_{d}$ are the original acceleration data and de-noised data, respectively.
We solve the problem (P2) in Equation (7) to estimate $\alpha_{d}$ by the optimal basis coefficients $\hat{\alpha}$ :

$$
\hat{\alpha}=\arg \min \|\tilde{\alpha}\|_{1} \text { such that }\|\boldsymbol{\Theta} \Psi \tilde{\alpha}-y\|_{2} \leq \varepsilon
$$

where $\varepsilon$ is an upper bound on the size of the noise contribution such that $\|e\|_{2} \leq \varepsilon$. Since $\|e\|_{2}=\|\boldsymbol{\Phi} \tilde{e}\|_{2} \leq$ $\|\boldsymbol{\Phi}\|_{2}\|\tilde{e}\|_{2}$, to set the threshold $\varepsilon$, the norm of the measurement noise $\|\tilde{e}\|_{2}$ must be estimated, such as by prior information or by making measurements with a sensor without CS. In the examples later, we chose $\varepsilon$ by minimizing the reconstruction error for a given number of measurements $m$ (i.e., a given $\mathrm{CR}$ ). In practice, one would not know the true signal $x$; so this optimum value of $\varepsilon$ is not available but we have found that the results are not sensitive to the choice of $\varepsilon$.

In this article, the discrete-time signal $x(t)$ shown in Figure 4 is used to investigate the application of CS to vibration signals used in SHM. In practice, if we want to acquire the data already in a compressed form, CS would be directly applied to the analog signal from the sensors; for example, since modern wireless accelerometers integrate the ADC into the sensor itself, CS would be included in the ADC. Kirolos et al. ${ }^{17}$ have developed 


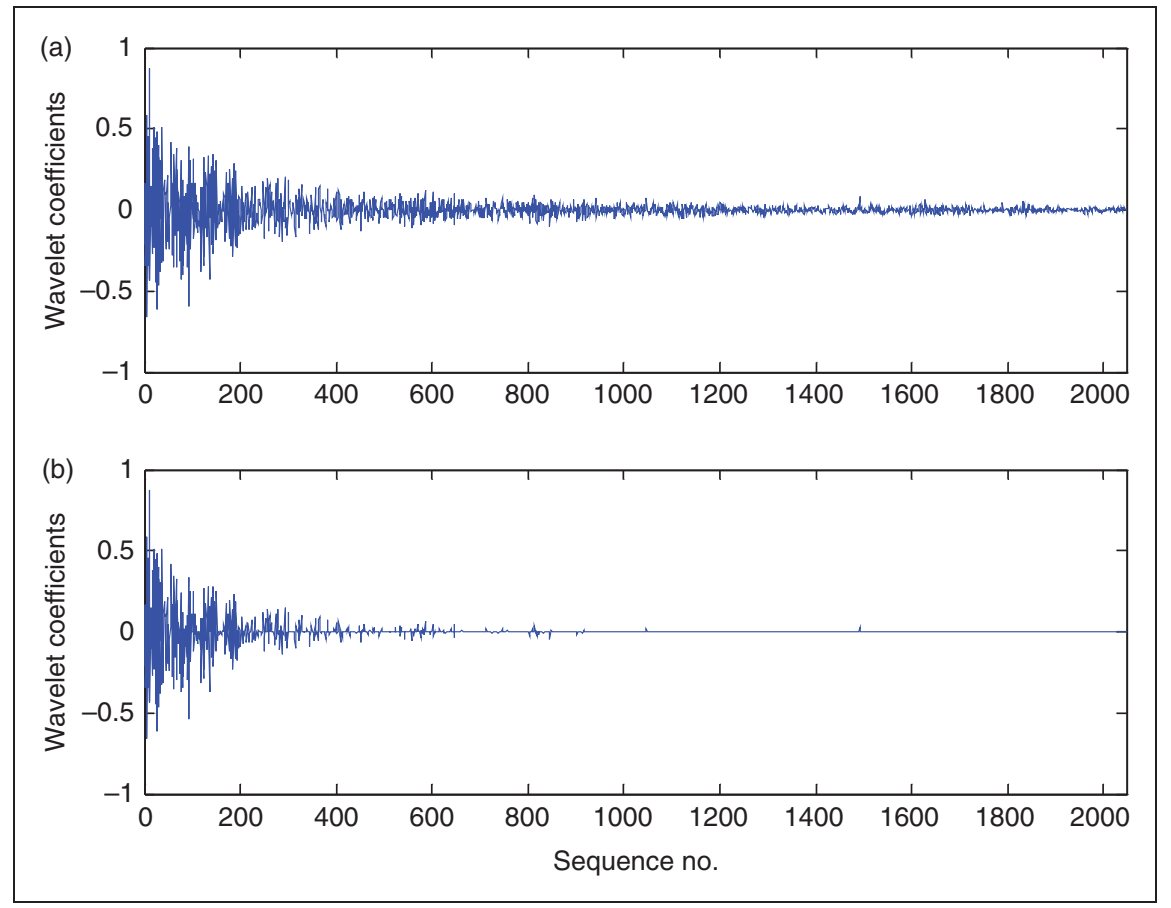

Figure 5. Wavelet coefficients of the acceleration data using the Haar wavelet basis: (a) original wavelet coefficients; (b) de-noised wavelet coefficients.

such an ADC that can implement the CS algorithm in a circuit. A CS sensor could be produced by integrating a wireless sensor with this ADC, although this has not yet been done commercially as far as we know.

\section{Compressive sampling results for Haar wavelet basis}

We used the $l_{1}$-magic package available online at http:// www.acm.caltech.edu/11magic/ to solve the CS problem (P2) in Equation (17) The Haar wavelet basis is selected to form the orthogonal basis matrix $\boldsymbol{\Psi}$. As noted earlier with reference to Figure 5, de-noising the wavelet coefficients reduces their number from $n=2048$ to $s=363$ nonzero wavelet coefficients. Here we examine the CS results for $m=2 s, m=3 s$, and $m=4 s$, which correspond to CRs of $n / m=2.82,1.88$, and 1.41 , respectively. The reconstruction error is calculated as:

$$
\xi=\frac{\|\hat{x}-x\|_{2}}{\|x\|_{2}}
$$

where $\hat{x}$ and $x$ are the CS reconstructed data and the original data, respectively. We also examine de-noised versions of the CS reconstructed data, where the reconstruction error of CS with de-noising (CSDN) is calculated as:

$$
\xi_{d}=\frac{\left\|\hat{x}_{d}-x_{d}\right\|_{2}}{\left\|x_{d}\right\|_{2}}
$$

where $\hat{x}_{d}$ and $x_{d}$ are the de-noised reconstructed data and de-noised original data.

The measurements of compressed data $y$ with $m=2 s, m=3 s$, and $m=4 s$ are shown in Figure 6. As in Equation (16), measurement vector $y$ is acquired by a linear projection $y=\boldsymbol{\Phi} x$, where $x$ is the original data and $\boldsymbol{\Phi}$ an $m \times n$ random matrix sampled from the normal distribution with zero mean and variance $1 / s$ The measurement matrix $\Phi$ can be built into the ADC, which means that the data can be acquired directly in a compressed form. The wavelet basis $\boldsymbol{\Psi}$ is not needed for acquiring the compressed data, and is only used when reconstructing (i.e., estimating) the original data.

The CS results for $m=2 s, m=3 s$, and $m=4 s$ are shown in Figures 7-9, respectively. Figure 7(e) and (f) compared with Figure 7(a) and (b) show that CS can approximately recover the data when $m=2 s$, but it is somewhat noisy. After de-noising the CS reconstructed data by a wavelet transform, the plots shown in Figure 7(g) and (h), and Figure 7(c) and (d) demonstrate that the CS de-noised data results are improved over the original and CS reconstructed data comparison. Figure 8 shows the reconstruction results with $m=3 s$, which look much better than the results with $m=2 s$ in Figure 7. Figure 9 shows the reconstruction results for $m=4 s$ lead to very good agreement with the original data and its de-noised version (compare Figure 9(a) and (c) with Figure 7(a) and (c), respectively). Clearly, 

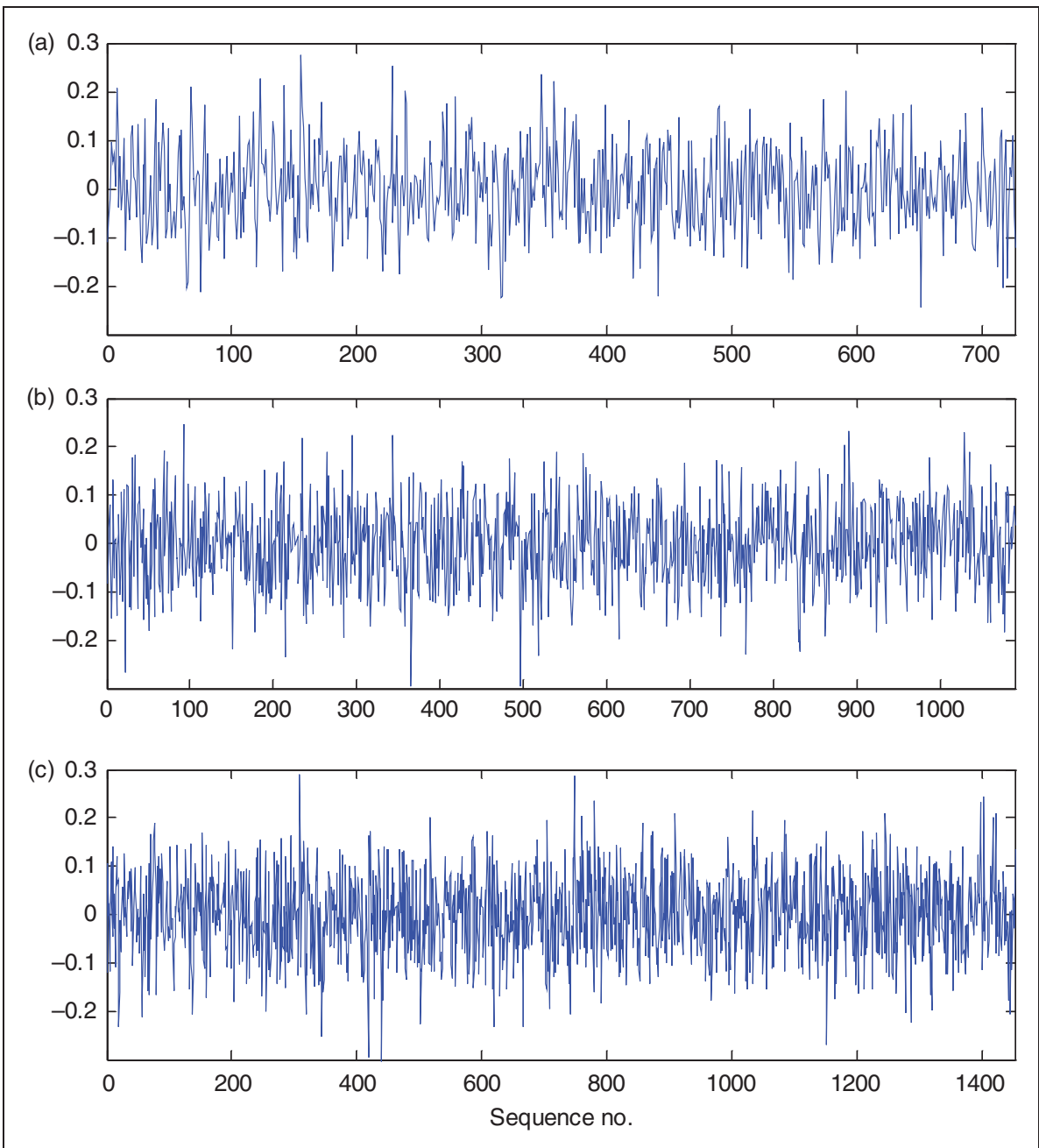

Figure 6. Measurements $y$ with: (a) $m=726$, (b) $m=1086$, and (c) $m=1452$.

the results are improved with increasing $m$, as expected. However, the goal of CS in this example is to compress the data and as expected, there is a trade-off between the accuracy of the reconstructed data and the amount of compression.

Figure 10 is the Fourier spectra of the original data and the reconstructed data. Figure 10(a) shows that the energy of the original data is mostly concentrated in the frequency interval from 0 to $10 \mathrm{~Hz}$. The Fourier spectra of the reconstructed data with $m=2 s=726$ are shown in Figure 10(c) and (d). There is a reduction in amplitude from the original data to the de-noised data. Figure 10(e) and (f) and Figure 10(g) and (h) show the Fourier spectra of the CS reconstructed data with $m=3 s$ and $m=4 s$, respectively. Clearly, the reconstruction results are better for large $m$; the reconstruction results for $m=3 s$ are acceptable, while for $m=4 s$ the results are much better.

\section{Comparison of compressive sampling with Fourier basis and wavelet basis}

In SHM, the Fourier and wavelet transforms are commonly used for vibration data processing. In this subsection, we make a comparison between the CS results with the Fourier basis and the Haar wavelet basis. To investigate the relation between reconstruction error and the amount of measurements using CS with these two bases, we choose a sequence of measurement numbers $m=2 s, 2.2 s, \ldots, 5.6 s$, where $s=363$. The orthonormal basis matrixes $\boldsymbol{\Psi}$ are formed by Fourier sinusoids and Haar wavelet basis, respectively. The results are shown in Figure 11, which shows that the CS reconstruction error with either basis decreases with an increase in the number of measurements, as expected. Also, Figure 11 shows that the reconstruction errors of CS with the wavelet basis are less than 

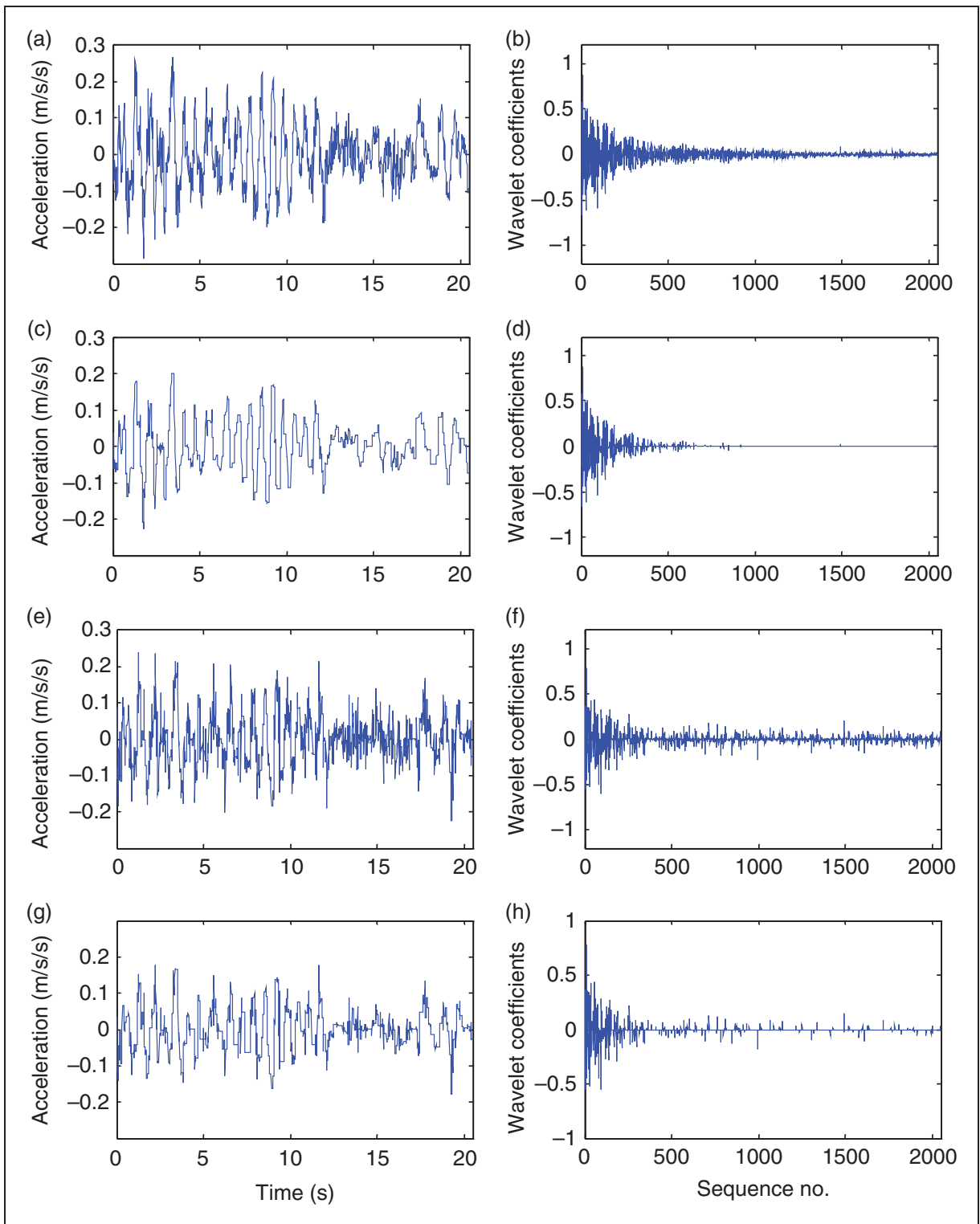

Figure 7. CS results of acceleration data: (a) original data $n=2048$; (b) original wavelet coefficients; (c) de-noised data of (a), the reconstruction error is $\xi=0.3525$; (d) de-noised wavelet coefficients of (b) used for (c), $s=363$; (e) CS reconstructed data, $m=2 s=726$, the reconstruction error is $\xi=0.5507$; (f) wavelet coefficients of CS reconstructed data; (g) de-noised data of (e), the reconstruction error is $\xi_{d}=0.4280$; (h) the de-noised wavelet coefficients of (f) used for $(\mathrm{g})$.

those with the Fourier basis with the same number of measurements.

\section{Comparisons with other data compression methods}

For comparison, data compression using the wavelet transform and Huffman coding are also used. In the field of image and video compression, the wavelet transform is widely applied, and it has been selected as the foundation for the new JPEG2000 imagery standard. $^{24}$ The main idea of wavelet-based data compression is that the analyzed signal is waveletdecomposed up to several scales or levels. After decomposition, most of the energy of the signal is concentrated into a small number of low-frequency wavelet coefficients. Then thresholding rules such as the hard or soft thresholding given earlier are used to filter the wavelet coefficients. The wavelet coefficients having a larger absolute value than the threshold are kept for reconstructing the signal back into the time domain, while the rest of the coefficients are zeroed. 

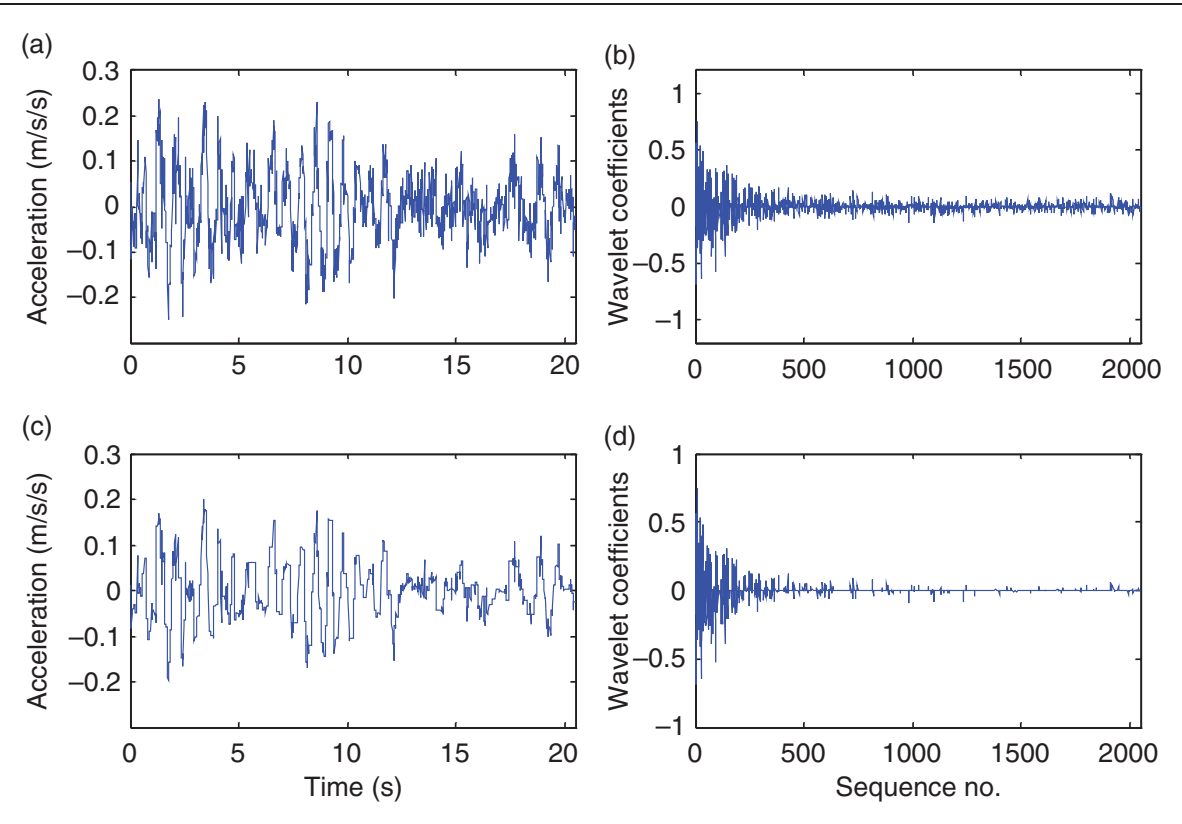

Figure 8. CS results of acceleration data with $m=3 s=1089$ : (a) CS reconstructed data, the reconstruction error is $\xi=0.3902$; (b) wavelet coefficients of (a); (c) de-noised data of (a), the reconstruction error is $\xi_{d}=0.2624$; (d) de-noised wavelet coefficients of (b) used for (c).
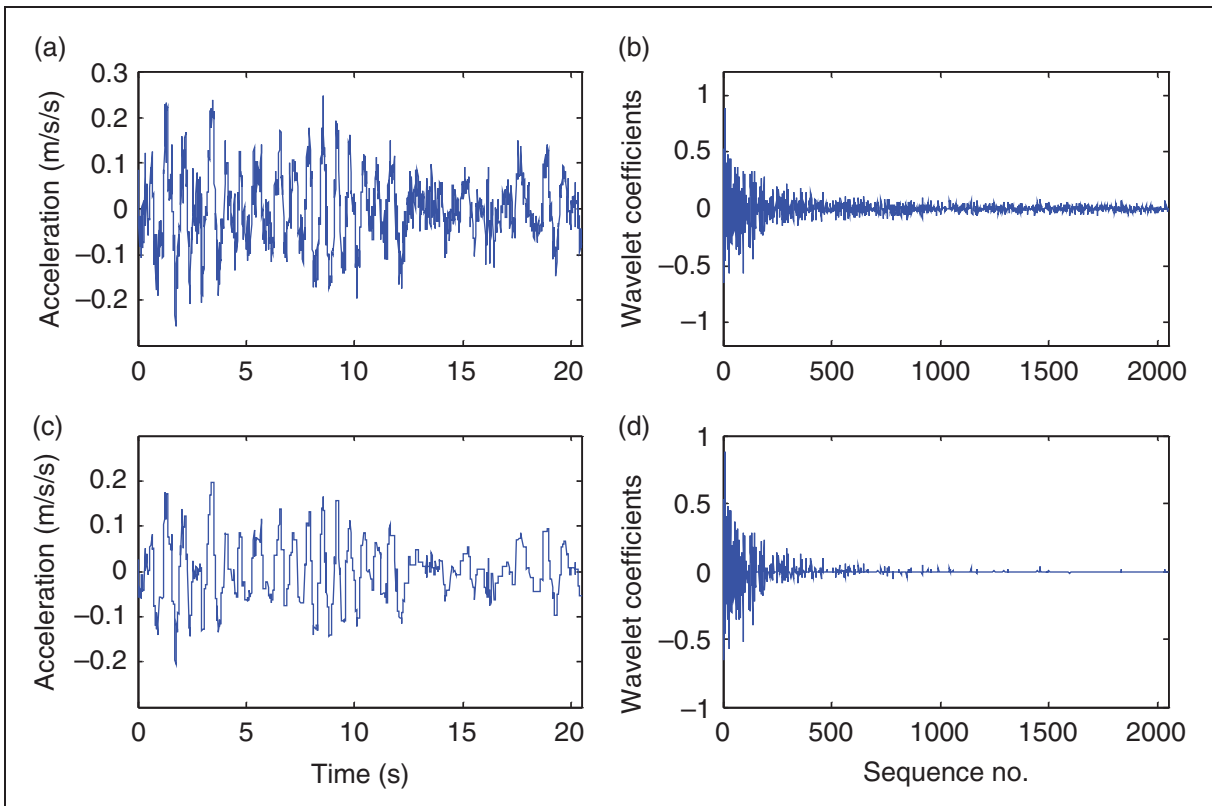

Figure 9. CS results of acceleration data with $m=4 s=1452$ : (a) CS reconstructed data, the reconstruction error is $\xi=0.272 \mathrm{I}$; (b) wavelet coefficients of (a); (c) de-noised data of (a), the reconstruction error is $\xi_{d}=0.165 \mathrm{I}$; (d) de-noised wavelet coefficients of (b) used for (c).

Therefore, only the nonzero coefficients and their locations need to be recorded and transmitted.

Huffman coding is a lossless compression technique. The main idea of Huffman coding is that the symbols occurring more frequently (i.e., have a higher probability of occurrence) are given shorter code words than the other less frequent symbols. ${ }^{24}$ Huffman coding can be classified into two different groups: static Huffman 

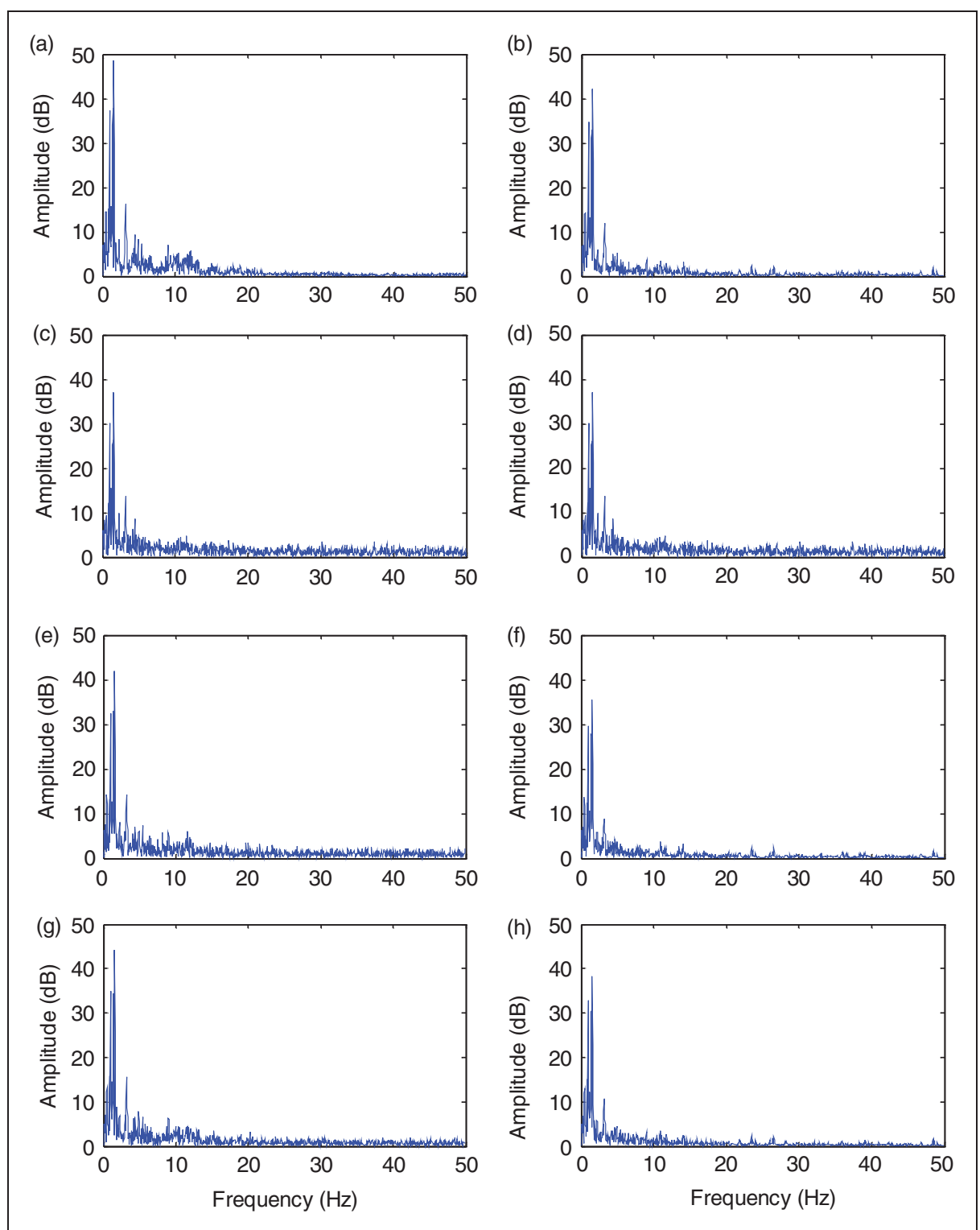

Figure 10. Fourier spectra of the original data and the reconstructed data: (a) original data, (b) de-noised data, (c) CS reconstruction $m=726$, (d) CSDN reconstruction $m=726$, (e) CS reconstruction $m=1089$, (f) CSDN reconstruction $m=1089$, (g) CS reconstruction $m=1452$, (h) CSDN reconstruction $m=1452$.

coding and adaptive Huffman coding. Static Huffman coding uses statistical symbol frequency tables, in which symbol frequencies are known in advance and the encoding table is sent to the decoder as part of the encoded file. Adaptive Huffman coding modifies the decoding table as characters are encoded, which allows the encoder to adapt to changing conditions in the input data. More details about the Huffman coding algorithm may be found in Sayood. ${ }^{25}$

The data compression results of CS, wavelet transform, and Huffman coding are shown in Table 1.
The data compression performance is measured using the CR:

$$
C R=\frac{N_{a}}{N_{c}}
$$

where $N_{a}$ and $N_{c}$ represent the original data size and compressed data size, respectively. Huffman coding methods include two stages. The first stage is decomposing the acceleration data using the wavelet transform and quantifying the wavelet coefficients by threshold. In the second stage, the wavelet coefficients 
are encoded with a binary code such as '100010010'. Both the static and adaptive Huffman coding methods are used in this example. The static Huffman coding records and transmits the statistical symbol frequency tables with the encoded stream of symbols, but the adaptive Huffman coding need not. Note that the adaptive Huffman coding CR shown in Table 1 is more than static Huffman coding. The wavelet transform method, without also using another encoding approach, only recodes the nonzero wavelet coefficients and their locations. The original acceleration data and wavelet coefficients are all accurate to four decimal digits. The location information of the wavelet coefficients is recorded as integers. All the data sizes are measured in bytes, and the data compression results are shown in Table 1. Table 1 shows that the CR of CS with wavelet basis is close to the static and adaptive Huffman coding methods and significantly less than the wavelet transform. Clearly, with respect to the $\mathrm{CR}$, the wavelet transform method is better than CS; it was found that it gives a length of compressed data that is only about twice the length of nonzero

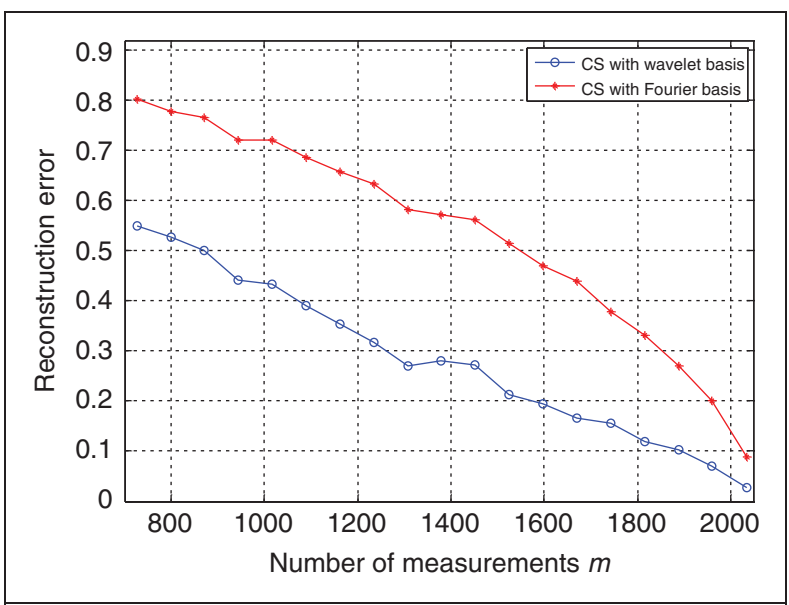

Figure II. Reconstruction error of CS with wavelet basis and Fourier basis. coefficients, but CS gives about three times the length of nonzero coefficients. With $m=3 s$, the reconstruction error $\xi$ of CS with the wavelet basis is about 0.3902 , which is close to the reconstruction errors of the wavelet transform and Huffman coding methods. However, with $m=3 s$, the reconstruction error $\xi$ of CS with the Fourier basis is 0.6872 which is much more than that for the other methods. Figure 12 shows the relation of $\mathrm{CR}$ and reconstruction error of CS, wavelet data compression, static Huffman coding, and adaptive Huffman coding methods. It can be seen from Figure 12 that the $\mathrm{CR}$ increases with an increase in reconstruction error for these four data compression methods. Clearly, with the same reconstruction error, the wavelet method has the best $\mathrm{CR}$, and CS with the wavelet basis is close to the Huffman coding methods for smaller reconstruction errors. The advantage of CS is that only the measurement matrix $\boldsymbol{\Phi}$ is needed when acquiring the data and this is independent of the choice of orthonormal basis $\boldsymbol{\Psi}$, which is only used when recovering the data. Thus, it is possible to design a physical sampling device that directly records compressed discrete data from the analog signal.

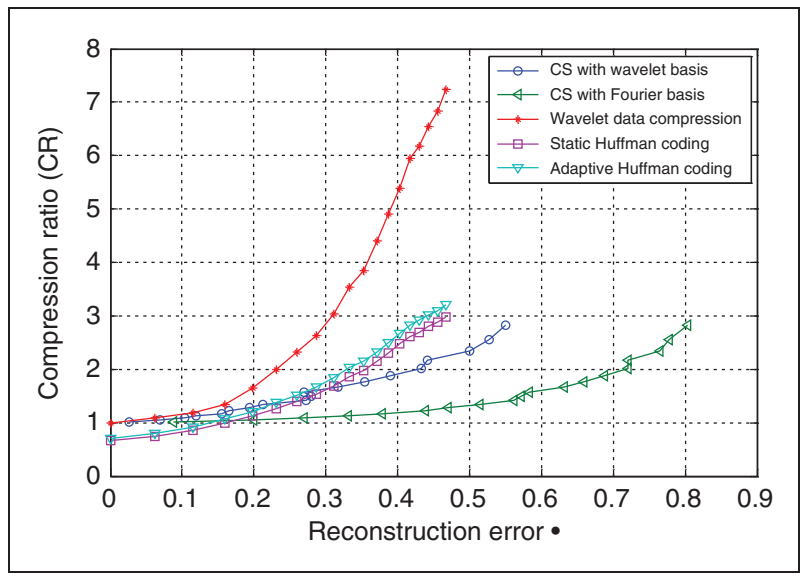

Figure 12. Relation of $C R$ and reconstruction error.

Table I. Comparison of various data compression methods

\begin{tabular}{|c|c|c|c|c|c|c|}
\hline & \multirow{2}{*}{ Original data } & \multirow{2}{*}{$\begin{array}{l}\text { CS with wavelet basis } \\
m=3 s\end{array}$} & \multirow{2}{*}{$\begin{array}{l}\text { CS with Fourier basis } \\
m=3 s\end{array}$} & \multirow{2}{*}{ Wavelet transform } & \multicolumn{2}{|c|}{ Huffman coding } \\
\hline & & & & & Static & Adaptive \\
\hline Data size (bytes) & 16,384 & 8712 & 8712 & 4256 & 8274 & 7631 \\
\hline CR & - & 1.88 & 1.88 & 3.85 & 1.98 & 2.15 \\
\hline Reconstruction error $\xi$ & - & 0.3902 & 0.6872 & 0.3525 & 0.3525 & 0.3525 \\
\hline
\end{tabular}




\section{Conclusions}

In this article, the potential of CS for compressing vibration data for SHM is investigated for the first time by using real acceleration data collected from the SHM system of the Shandong Binzhou Yellow River Highway Bridge. Since CS sensors are not yet commercially available, we digitally simulate their behavior. The results show that the values of CRs achieved by CS are not high because these data are not naturally sparse in the wavelet basis. The sparseness of the transformed signal for the chosen basis controls the applicability of CS, since greater sparseness means that CS can compress the data better. The comparison of CS results using the Fourier basis and the wavelet basis demonstrates that the latter works better than the former when applying CS to acceleration signals. Although the CR shown in this work is not better than that the existing wavelet-based data compression method, CS can be designed into a physical device such as an ADC to acquire the data directly in a compressed form, in contrast to the traditional data compression methods. In the studies in future, we will explore a Bayesian statistical approach for ill-conditioned inverse problems that should allow better control of the relation between compression ratios and reconstruction errors.

\section{Acknowledgments}

One of the authors (Yuequan Bao) acknowledges the support provided by the China Scholarship Council while he was a visiting student researcher at the California Institute of Technology. This research is also supported by grants from National Natural Science Foundation of China (NSFC grant nos. 50538020, 50278029, and 50525823) and the Science and Technology Program for the West Communication Construction of the Ministry of Construction, China, which supported the first and third authors (Yuequan Bao and Hui Li).

\section{References}

1. Ko JM. Health monitoring and intelligent control of cable-supported bridges. In: Proceedings of the International Workshop on Advanced Sensors, Structural Health Monitoring and Smart Structures, Keio University, Tokyo, Japan, 10-11 November 2003.

2. Zhang $\mathrm{Y}$ and $\mathrm{Li}$ J. Wavelet-based vibration sensor data compression technique for civil infrastructure condition monitoring. J Comput Civil Eng 2006; 20(6): 390-399.

3. Lynch JP and Loh KJ. A summary review of wireless sensors and sensor networks for structural health monitoring. Shock Vibration Digest 2005; 38(2): 91-128.
4. Spencer Jr BF, Ruiz-Sandoval M, and Kurata N. Smart sensing technology: Opportunities and challenges. Struct Contr Health Monitor 2004; 11: 349-368.

5. Xu N, Rangwala S, Chintalapudi K, Ganesan D, Broad A, Govindan R, and Estrin D. A wireless sensor network for structural monitoring. In: Proceedings of the ACM Conference on Embedded Networked Sensor Systems. Baltimore, MD, USA, November 2004.

6. Lynch JP, Sundararajan A, Law KH, Kiremidjian AS, and Carryer E. Power-efficient data management for a wireless structural monitoring system. In: Proceedings of the 4th International Workshop on Structural Health Monitoring, Stanford, CA, 15-17 September 2003, pp.1177-1184.

7. Peterson CV, and Hutt CR. Lossless compression of seismic data. In: Proceedings of the 26th Asilomar Conference on Signals, Systems, and Computers, Pacific Grove, CA, Vol. 2, 26-28 October, 1992, p.712-716.

8. Kiely AB, and Pollara F. A seismic data compression system using subband coding. TDA Progress Report No. 42-121, 1995.

9. Spanias AS, Jonsson SB, and Stearns SD. Transform methods for seismic data compression. IEEE Trans Geosci Remote Sensing 1991; 29: 407-416.

10. Candès EJ. Compressive sampling. In: Proceedings of the International Congress of Mathematicians, Madrid, Spain, 2006, p.1433-1452.

11. Candès EJ and Tao T. Decoding by linear programming. IEEE Trans Inform Theory 2005; 51: 4203-4215.

12. Candès EJ, Romberg J, and Tao T. Robust uncertainty principles: Exact signal reconstruction from highly incomplete frequency information. IEEE Trans Inform Theory 2006; 52(2): 489-509.

13. Donoho D. Compressed sensing. IEEE Trans Inform Theory 2006; 52(4): 1289-1306.

14. Baraniuk R. A lecture on compressive sensing. IEEE Signal Process Mag 2007; 24(4): 118-121.

15. Takhar D, Laska JN, Wakin MB, Duarte MF, Baron D, Sarvotham S, et al. A new compressive imaging camera architecture using optical-domain compression. In: Proceedings of the Computational Imaging IV at SPIE Electronic Imaging, San Jose, California, January 2006.

16. Bajwa WU, Haupt J, Sayeed AM, and Nowak R. Compressive wireless sensing. In: Proceedings of the 5th International Conference on Information Processing in Sensor Networks (IPSN'06), Nashville, TN, 2006, pp.134-142.

17. Kirolos S, Laska J, Wakin M, Duarte M, Baron D, Ragheb T, et al. Analog-to-information conversion via random demodulation. In: Proceedings of the IEEE Dallas Circuits and Systems Workshop, Dallas, Texas, October 2006.

18. Baraniuk RG, Davenport M, DeVore R, and Wakin MB. A simple proof of the restricted isometry property for random matrices. Construct Approxim 2008; 28(3): 253-263.

19. Daubechies I. Ten lectures on wavelets. Philadelphia: SIAM, 1992. 
20. Meyer Y. Wavelets and operators. Cambridge: Cambridge University Press, 1992.

21. Mallat S. A wavelet tour of signal processing. 2nd ed. San Diego, CA: Academic Press, 1999.

22. Donoho DL and Johnstone IM. Ideal spatial adaptation by wavelet shrinkage. Biometrika 1994; 81: 425-455.

23. Li H, Ou JP, Zhao XF, Zhou WS, Li HW, and Zhou Z. Structural health monitoring system for the Shandong
Binzhou Yellow River Highway Bridge. Comput-aided Civil Infrastruct Eng 2006; 21: 306-317.

24. Usevitch BE. A tutorial on modern lossy wavelet image compression: Foundations of JPEG 2000. IEEE Signal Process Mag 2001; 18(5): 22-35.

25. Sayood K. Introduction to data compression. 3rd ed. San Francisco, CA, USA: Morgan Kaufmann Publishers, 2000. 